\title{
Essais
}

Revue interdisciplinaire d'Humanités

$7 \mid 2015$

Normes communiquées, normes communicantes

\section{Jean-Pierre Meunier, Des images et des mots: Cognition et réflexivité dans la communication}

\section{Mélanie Mauvoisin}

\section{(2) OpenEdition}

1 Journals

Édition électronique

URL : http://journals.openedition.org/essais/6565

DOI : $10.4000 /$ essais. 6565

ISSN : 2276-0970

Éditeur

École doctorale Montaigne Humanités

\section{Édition imprimée}

Date de publication : 1 décembre 2015

Pagination : 156-158

ISBN : 978-2-9544269-6-9

ISSN : 2417-4211

Référence électronique

Mélanie Mauvoisin, « Jean-Pierre Meunier, Des images et des mots : Cognition et réflexivité dans la communication », Essais [En ligne], 7| 2015, mis en ligne le 26 février 2021, consulté le 28 février 2021. URL : http://journals.openedition.org/essais/6565; DOI : https://doi.org/10.4000/essais. 6565 


\section{Comptes rendus}

Jean-Pierre Meunier, Des images et des mots : Cognition et réflexivité dans
la communication. Louvain-La-Neuve, Éditions Academia, 2013, 280 p.

Cet ouvrage du sémiologue, spécialiste des théories générales de la communication, Jean-Pierre Meunier, propose de poursuivre le projet de Saussure qui désirait, dans le prolongement de la linguistique, faire émerger une nouvelle science, plus grande encore, à travers la sémiologie. L'auteur s'engage ainsi à aller plus loin et à mettre en exergue, comme le titre du livre l'indique, le rôle majeur de la cognition et de la réflexivité dans les relations de communication. Il s'intéresse alors à une question qui passionne de nombreuses disciplines universitaires (linguistique, psychologie cognitive, sociologie, Sciences de l'Information et de la Communication, philosophie, etc.), celle de notre rapport au langage à travers les images et les mots. Son objectif est ainsi de développer ce qui constitue un nouveau point de vue théorique et qui s'appuie plus particulièrement sur les représentations mentales et les opérations cognitives accompagnant la communication.

Ce livre se divise en six grandes parties à partir desquelles l'auteur entend montrer l'évolution et les caractéristiques de notre système cognitif et de notre réflexivité à partir des processus communicationnels et d'exemples différents qui viennent étayer son propos. Les deux premières parties sont consacrées pour une grande part à l'élaboration d'une revue de l'évolution historique de la sémiologie au profit notamment des chercheurs non-initiés. Les rappels constants des exemples et des méthodes citées particulièrement en introduction mettent en exergue la volonté de l'auteur de clarifier et de faire émerger une logique de sens quant à la relation qu'il entend mettre en avant entre sémiologie et cognition.

Le sémiologue propose ainsi au début de son livre de rappeler quelques clés de compréhension de la sémiologie au travers d'une introduction dans laquelle il revient sur l'histoire mais aussi les méthodes et les grands auteurs 
de la discipline. Dans cette volonté d'explicitation, l'auteur aborde une approche phénoménologique des phénomènes vécus durant les rapports de communication et s'interroge tout le long du livre sur l'expérience subjective dans le contexte de cognition. À partir des différents mécanismes de cognition (mimétisme, comparaison, analogie, métaphore, schémas et schématisation, intégration conceptuelle ou encore abduction), l'auteur revient dans un premier chapitre sur les "opérations cognitives de base ». Il part alors du postulat que le mimétisme est l'aptitude de base de la cognition humaine, c'est-à-dire le fondement de toute relation. Le sémiologue rappelle ainsi le rôle fondamental du corps dans l'expérience que celui-ci fait de son environnement et dans son rapport aux autres. Selon lui, " c'est le corps, avec cette capacité innée à entrer en résonance avec tout ce qu'il perçoit de son environnement qui constitue (...) la base de la cognition » (p. 29). C'est donc après avoir expliqué l'importance du mimétisme dans la cognition et les relations sociales, qu'il va décrire chacun des mécanismes cités plus haut en prenant soin de les illustrer d'un exemple concret et le plus souvent, par le biais d'une analyse publicitaire.

Dans le troisième chapitre, portant sur les médiations sémiotiques que sont l'image et le langage verbal, le chercheur nuance son propos. Bien que la cognition humaine soit fortement iconique, l'auteur explique cependant qu'il ne faut pas limiter la cognition à un simple effort inconscient de projection métaphorique. Au contraire, il semblerait qu'un effort supplémentaire d'attention soit nécessaire à " l'élaboration mentale » de la compréhension et que celui-ci s'appliquerait à des niveaux différents selon certains mécanismes de cognition. Ces médiations sémiotiques, que sont les images et les verbes, sont en effet rattachées à une culture où chacune des "interférences " et des " interactions " avec les opérations cognitives sont essentielles à la " pensée implicite " (p. 62). En d'autres termes, elles peuvent également être considérées comme des médiations sociales. En reprenant, entre autres, l'exemple de la célèbre publicité pour les pâtes Panzani (p. 79), l'auteur développe la théorie de Roland Barthes et explique qu'il faut aller au-delà des simples " codes " proposés par la connotation. Selon lui, il existerait des "scénarios ", plus ou moins " cadrés " surtout dans les publicités. Ceux-ci seraient alors ce qui permettrait d'appréhender la publicité à travers un affaiblissement voire une élimination des catégories abstraites dont font partie certains éléments de l'image, pour mieux renforcer d'autres scénarios, comme ici la cuisine et les saveurs.

Le quatrième chapitre, intitulé " cognition et réflexivité langagière ", constitue l'une des parties les plus techniques du livre. L'enjeu y est de comprendre " ce que la distanciation, la réflexivité et la discrétisation des représentations qu'entraîne la face signifiante du signe linguistique impliquent sur le plan de l'organisation et la dynamique des représentations mentales " (p. 147). Pour lui, il semble indéniable que la réflexivité dans le discours se distingue selon des degrés différents. Lauteur, poursuivant cette visée explicative, propose ainsi en fin de chapitre une tentative de catégorisation 
globale de la communication langagière selon les différents niveaux de réflexion intersubjective. Puis, dans la lignée de sa réflexion sur les médiations sémiotiques, Jean-Pierre Meunier intègre, dans le cinquième chapitre, les techniques et les technologies de communication à travers son analyse. Son intérêt est alors de voir l'importance des effets que peuvent avoir celles-ci sur les opérations cognitives imaginatives et langagières. Il aborde ainsi et met en exergue un rapport aujourd'hui plus que jamais inévitable entre les instruments de communication et les opérations cognitives qui prennent forme à travers eux.

Le dernier chapitre vient clore ce long travail d'explicitation en mettant en avant l'interaction complexe entre les images et les mots. Ici, l'auteur se focalise sur les formes de langage. Qu'il soit imagé ou verbal, le langage semble constituer un moyen de différencier le "plan cognitif "à tel point que chacun peut avoir, réciproquement, un effet sur l'autre. Les combinaisons imageverbe sont illimitées mais certaines ont un niveau d'analyse plus complexe qui peut parfois même être difficile à appréhender. On notera les exemples cités par l'auteur : les photographies de presse ou d'exposition, les hypermédias qui mettent en réseau des images, des textes et des vidéos, les musées, etc. Mais on pourrait également ajouter à cette liste l'ensemble des technologies mobiles.

En définitive, dans son ouvrage, Jean-Pierre Meunier souhaite par son approche mettre en avant la relation entre le mimétisme et la réflexivité qui constitue selon lui un élément important dans toute recherche en Communication sur les rapports entre émetteur(s) et destinataire(s). Son livre tente de montrer en quoi l'acquisition du langage est un enjeu essentiel à la fois du point de vue de la structure de nos représentations, mais également dans la manière dont celle-ci contribue à modifier notre rapport au monde. Le langage est donc ce qui permet d'acquérir un psychisme autoréflexif. Il est ce qui nous permet d'appréhender, chacun à partir de ses propres connaissances et de ses expériences vécues par le biais du mimétisme, les rapports de communication au quotidien. Or, bien que les exemples de l'auteur soient intéressants et parfaitement choisis, on pourra regretter le manque d'innovation relatif à certaines perspectives mises en jeu à travers l'ouvrage (notamment dans le cinquième chapitre portant sur les médiations techno-sémiotiques), ainsi que leur caractère ponctuellement redondant. Cependant, et malgré ces remarques, c'est bien par sa façon de penser la relation entre l'image et les mots, que la lecture de l'œuvre de Jean-Pierre Meunier s'avère particulièrement passionnante pour les chercheurs et doctorants qui s'intéressent à l'évolution contemporaine des théories générales de la Communication.

Mélanie Mauvoisin

EA 4426 MICA

Université Bordeaux Montaigne melanie.mauvoisin@etu.u-bordeaux3.fr 